\title{
The Problem of Equilibrium Processes in Thermodynamics
}

\author{
David A. Lavis \\ King's College London, Department of Mathematics, Strand, London WC2R 2LS, U.K. \\ To appear in: Studies in History and Philosophy of Modern Physics
}

\begin{abstract}
It is well-known that the invocation of 'equilibrium processes' in thermodynamics is oxymoronic. However, their prevalence and utility, particularly in elementary accounts, presents a problem. We consider a way in which their role can be played by sets of sequences of processes demarcated by curves carrying the property of accessibility. We also examine the vexed question of whether equilibrium processes are necessarily reversible and the revision of this property in relation to sets of sequences of such processes.
\end{abstract}

Keywords: controllability, achievability, accessibility, recoverability, reversibility

\section{Introduction}

The systems of classical thermodynamics - that is to say equilibrium thermodynamics, as distinct from various possible extensions to non-equilibrium situations - have no spontaneous behaviour. The states of the system are equilibrium states and the space $\Xi$ of these states is a thermodynamic system. All transitions between states, called processes, are a result of an outside intervention using a set of control variables. As Wallace (2014) points out the name for the study of systems with this character is control theory and the question to be asked is: Given the system is in a particular state, can the control variables be manipulated to bring the system into another specified state?

It is convenient to use the symbol $\Xi$ to denote both the thermodynamic system and its space of states. In the latter sense $\Xi$ is an open convex set in $\mathbb{R}^{n+1}$ for some integer $n>0$. The elements of the state-vector $\mathbf{x} \in \Xi$ are extensive variables. Those of mechanical type are referred to as deformation variables, with each being associated with an intensive control variable. Examples are the volume of a fluid with associated control variable being the pressure exerted by the force on a piston and the magnetic moment of a magnet controlled by an applied magnetic field. ${ }^{1}$

The characteristic feature of a thermodynamic as distinct from a mechanical system is the presence of at least one thermal variable. A system with exactly one thermal variable is called $\boldsymbol{s i m p l e}^{2}$ and that one thermal variable can be identified with the internal energy $U{ }^{3}$ We shall, henceforth, suppose that the system in question is simple; so to be specific $\boldsymbol{x}:=\left(\boldsymbol{x}^{\mathrm{T}}, \boldsymbol{x}^{\mathrm{D}}\right)$, where $\boldsymbol{\chi}^{\mathrm{T}}:=U$ and $\boldsymbol{x}^{\mathrm{D}}$ is an $n$-dimensional vector of deformation variables. A thermodynamic process $\mathbf{x} \rightarrow \mathbf{x}^{\prime}$ is a manipulation of the control variables to change the state of the system from $\mathbf{x}$ to $\mathbf{x}^{\prime}$. For this statement to make sense we must assume:

\footnotetext{
Email address: david.lavis@kcl.ac.uk (David A. Lavis)

${ }^{1}$ And it is, of course, the case that, once the equations of state relating the extensive and intensive variables are known, the state of the system can be specified in a state space coordinated by a mixture of extensive and intensive variables.

${ }^{2}$ In the account of thermodynamics by Lieb and Yngvason (1999) a simple system has this property together with a number of additional properties (op. cit. Sect. 3), which do not concern us at this stage.

${ }^{3}$ The thermal variable could be identified as the entropy $S$, but in most accounts that is a derived quantity appearing later in the analysis.
} 
The Hypothesis of Controllability: that all interactions between the system and its environment are controllable. This includes not just manipulations of the control variables associated with the deformation variables but also all other means by which the internal energy can be changed.

The Hypothesis of Achievability: that a possible process is achievable, exactly in a finite amount of time (which will normally include a final 'leave-it-alone' stage, Wallace, 2014) by a purposeful manipulation of the control variables.

A number of points are of note:

(i) These hypotheses encompass the minus first law of thermodynamics of Brown and Uffink (2001). ${ }^{4}$

(ii) Although, as we shall see, the work of this paper has similarities with that of Norton (2016), a significant difference between us is his (implicit) rejection of the hypothesis of achievability. ${ }^{5}$

(iii) The existence of a process $\boldsymbol{x} \rightarrow \boldsymbol{x}^{\prime}$ does not imply the passage along a sequence of (equilibrium) states in $\Xi$ from $\boldsymbol{x}$ to $\boldsymbol{x}^{\prime}$. With some exceptions (e.g. Giles, 1964) accounts of classical thermodynamics restrict, as we have indicated, the states of the system to equilibrium states, meaning that the only defined states of a process are its end points.

(iv) As a consequence of (iii), a process is specified in terms of its end points together with a description of the manipulations of the control variables used to bring it about.

(v) As a consequence of (iv), there will in general be many different processes denoted by $\boldsymbol{x} \rightarrow \boldsymbol{x}^{\prime}$. Thus a useful concept is that of accessibility (Buchdahl, 1966; Lieb and Yngvason, 1999). The state $\boldsymbol{x}^{\prime}$ is accessible from $\boldsymbol{x}$, written $\boldsymbol{x} \prec \boldsymbol{x}^{\prime}$ if there is at least one process $\boldsymbol{x} \rightarrow \boldsymbol{x}^{\prime}$.

Accessibility $\boldsymbol{x} \prec \boldsymbol{x}^{\prime}$ can be unqualified, meaning that there exists at least one among all the possible manipulations of the control variables which can be employed to produce a process $\boldsymbol{x} \rightarrow \boldsymbol{x}^{\prime}$, or qualified, meaning that only certain manipulations are allowed. The case of importance in the latter category is the implementation of an adiabatic process as described in Sect. 2.1.1. Buchdahl and Lieb and Yngvason consider only adiabatic accessibility, for which they use the symbol ' $\prec$ '. We shall begin by considering unqualified accessibility using ' $\prec$ '. If $\boldsymbol{x} \prec \boldsymbol{x}^{\prime}$ and $\boldsymbol{x}^{\prime} \prec \boldsymbol{x}$ then $\boldsymbol{x}$ is said to be recoverable from $\boldsymbol{x}^{\prime}$ (and vice-versa), ${ }^{6}$ denoted as $\boldsymbol{x} \succ \boldsymbol{x}^{\prime}$, with $\boldsymbol{x} \prec \boldsymbol{x}^{\prime}$ asserting that $\boldsymbol{x}$ is irrecoverable from $\boldsymbol{x}^{\prime}$; that is $\boldsymbol{x} \prec \boldsymbol{x}^{\prime}$ but not $\boldsymbol{x}^{\prime} \prec \mathbf{x}$. When we need to discuss adiabatic accessibility, recoverability and irrecoverability we use ' ${ }^{\mathrm{A}}$ ', ' $\stackrel{\mathrm{A}}{\gtrless}$ ' and ' $\gtrless^{\mathrm{A}}$ ', respectively.

In Sect. 2 we discuss the weaknesses of the standard definition of an equilibrium process along a curve in $\Xi$, emphasising the distinction between this and the question as to whether the process is reversible, and in Sect. 2.1 we propose replacement definitions for both of these based on accessibility. Sects. 2.1.1 and 2.1.2 apply these new definitions to adiabatic and isothermal processes, respectively and Sects. 3.1 and 3.2 discuss the cases of a perfect fluid and a cycle of processes. We compare and contrast our account with that of Norton (2016) in Sect. 4 and our conclusions are contained in Sect. 5.

\footnotetext{
${ }^{4}$ They argue that none of the laws of thermodynamics actually asserts that a system not in equilibrium attains an equilibrium state and that it does so must constitute an addition law.

${ }^{5}$ This is discussed in Sect. 5.

${ }^{6}$ The minefield associated with the various uses of the term 'reversible' in thermodynamics is carefully negotiated by Uffink (2001). He points out that some confusion is generated in the English translations of the writings of Planck and Clausius where the terms umkehrbarheit and reversibel are conflated to the single word 'reversible'. He recommends the use of the term recoverable when "the only thing that counts is the retrieval of the initial state" (ibid, p. 316). Following this advice we restrict the use of the term 'reversible' to situations where, within the development of a picture of an equilibrium process, the path of the process is reversed.
} 


\section{Equilibrium Processes}

At the outset there is a problem of terminology in the intimate and often confusing relationships between:

(a) a quasi-static process,

(b) an equilibrium process,

(c) a reversible process.

This is compounded by the profusion of overlapping and sometimes contradictory definitions of what is meant by a 'quasi-static process', ${ }^{7}$ containing as they do both a reference to what such a process is and how it is implemented. Thus we read that quasi-static processes are "those that may be considered as a sequence of neighbouring equilibrium states" (Lebon et al., 2008, p. 4) and that "a quasi-static process is a change in the state of the system that is conducted infinitesimally slowly such that, at each instant, the system is in thermodynamic equilibrium with its environment, and its thermodynamic properties [‥] remain welldefined throughout the process" (Samiullah, 2007, p. 608). Taken together we may infer from these quotes that a quasi-static process is just an equilibrium process together with some gloss as to how this process may be carried out. So, for the sake of discussion, let us agree to take 'equilibrium process' and 'quasi-static process' as synonyms and pass to the more interesting relationship between (b) an equilibrium process, and (c) a reversible process. Norton (2016, p. 43) refers to "thermodynamically reversible or quasi-static processes" at the outset of his paper and tends throughout to treat them as synonyms. ${ }^{8}$ While, as we shall argue, reversibility (as distinct from recoverability) is a useful description only for equilibrium processes (and our replacement thereof) the converse is by no means obvious. Thus, for example, Buchdahl (1966, pp. 52-54) gives a proof of the reversibility of quasi-static processes and MacDonald (1995, p. 1122) gives an example of a quasi-static irreversible process. In the interests of clarity it seems important to keep separate the question of the replacement for equilibrium processes and the second question as to whether, and in what sense, they can be regarded as reversible.

Let $\mathcal{L}\left(\mathbf{x}_{0}, \mathbf{x}_{1}\right)$ be a simple, directed and continuous curve in $\Xi$ parameterized by $\mathbf{x}=\mathbf{x}(\lambda)$, for $\lambda \in[0,1]$, with $\mathbf{x}(0):=\mathbf{x}_{0}$ and $\mathbf{x}(1):=\mathbf{x}_{1}$. The curve parameterized in the reverse direction is denoted by $\mathcal{L}\left(\mathbf{x}_{1}, \mathbf{x}_{0}\right)$.

Definition 1: $\mathcal{L}\left(\mathbf{x}_{0}, \boldsymbol{x}_{1}\right)$ is an accessible curve if $\mathbf{x}(\lambda) \prec \mathbf{x}\left(\lambda^{\prime}\right), \forall 0 \leq \lambda<\lambda^{\prime} \leq 1$.

It should be emphasised that just as accessibility can be unqualified or qualified, an accessible curve (that is to say the property of having accessibility between all directed pairs on the curve) can also be unqualified or qualified. And it is, of course, the case that a particular curve may be piecewise divisible into parts having different types of accessibility. In particular we shall be concerned with the case where $\mathcal{L}\left(\mathbf{x}_{0}, \mathbf{x}_{1}\right)$ is an adiabatically accessible curve and situations where the curve may be accessible but not adiabatically accessible.

Consider now the:

\section{Definitions 2:}

2-1: An equilibrium process along the curve $\mathcal{L}\left(\mathbf{x}_{0}, \mathbf{x}_{1}\right)$

$$
\mathfrak{E}\left(\mathcal{L}\left(\mathbf{x}_{0}, \mathbf{x}_{1}\right)\right):=\left\{\begin{array}{l}
\text { By some prescribed manipulation of the control variables } \\
\text { the state of the system passes along } \mathcal{L}\left(\mathbf{x}_{0}, \mathbf{x}_{1}\right) \text { from } \mathbf{x}_{0} \text { to } \mathbf{x}_{1} .
\end{array}\right\} .
$$

2-2: An equilibrium process $\mathfrak{E}\left(\mathcal{L}\left(\mathbf{x}_{0}, \mathbf{x}_{1}\right)\right)$ is reversible if the process $\mathfrak{E}\left(\mathcal{L}\left(\mathbf{x}_{1}, \mathbf{x}_{0}\right)\right)$, using the same control variables, also exists.

\footnotetext{
${ }^{7}$ The literature for this is extensively documented and analysed by Norton (2016). In consequence our comments are rather brief.

${ }^{8}$ Although, in Sect. 7.9, he does discuss both reversible and irreversible equilibrium processes, giving as an example the case of a perfect fluid where, along a curve in the thermodynamic space, there can be both a reversible and a "fully irreversible" process.
} 
Without prejudice as to whether these definitions are viable it is clear that a necessary condition for an equilibrium process along $\mathcal{L}\left(\mathbf{x}_{0}, \mathbf{x}_{1}\right)$ is that the curve is accessible and the equilibrium process will be reversible only if both $\mathcal{L}\left(\mathbf{x}_{0}, \mathbf{x}_{1}\right)$ and $\mathcal{L}\left(\mathbf{x}_{1}, \mathbf{x}_{0}\right)$ are so accessible.

$2-1$ is the definition of an equilibrium process in most texts on thermodynamics; often taking (Pippard, 1961 , p. 30) differential forms in the extensive variables as instances of infinitesimal equilibrium processes. But a process involving the application of control variables will drive the system from equilibrium with the return to an equilibrium state occurring only after the 'leave-it-alone' stage which allows it to settle down. Thus equilibrium processes are (Cooper, 1967, p. 174) "either a contradiction in terms or limits of processes through non-equilibrium states which cannot be described in terms of equilibrium theory." Rather more forcefully Norton (2016, p. 43-44) observes that:

Incantations of "infinitely slow", "insensible" and "infinitesimal' have no magical powers that overturn the law of the excluded middle. Either a system is in equilibrium or it is not; it cannot be both.

In Part II of his paper Norton undertakes an exhaustive analysis of the way that this problem is treated (or elided) in the literature of thermodynamics, supporting his contention (ibid, p. 45, our italics) that: ${ }^{9}$

The label "thermodynamically reversible process" denotes a set of irreversible processes in a thermal system, delimited by the set of equilibrium states.

It is also worth noting his discovery that Duhem adopted a similar approach. Thus:

Cette suite d'états d'équilibre $\alpha, \beta, \gamma, \delta, \ldots$ qui n'est parcourue dans aucune modification réelle du système, est, en quelque sorte, la frontière commune des modifications réelles qui conduisent le système de l'état 1 à l'état 2. (Duhem, 1902, p. 78)

Norton proposes a particular reconception of equilibrium processes based on the perception contained in these two quotations. We shall develop a different account, also based on curves in $\Xi$. In both Norton's and our approaches the role of the curve $\mathcal{L}\left(\mathbf{x}_{0}, \mathbf{x}_{1}\right)$ is one of delimiting, or synonymously of demarcating, a set of processes. For the sake of the discussion, we shall call our proposed relationship between processes and $\mathcal{L}\left(\mathbf{x}_{0}, \mathbf{x}_{1}\right)$ demarcation, allowing reservation of the term delimitation ${ }^{10}$ to the corresponding relationship proposed by Norton. The rest of this section describes the relationship of demarcation and, following examples in Sect. 3, Norton's account of delimitation is described and contrasted with demarcation in Sect. 4 .

At this point it is worth noting one common feature of both the approach proposed in this paper and that of Norton. Arising from our account so far it is clear that a thermodynamic system acted on by control variables cannot always be in a thermodynamic equilibrium state identified with a point in $\Xi$. Norton is not alone (see e.g. Landsberg 1956, p. 367; Callen 1985, p. 96) in saying that the system is then in a 'nonequilibrium state', although as pointed out by Buchdahl $(1966$, p. 10) "this notion is [...] meaningless, for when [the system] is not in equilibrium some of the [thermodynamic variables] have no well-defined values." This last assertion excepts the case where, as in Giles (1964), the space of states is extended in some way to include non-equilibrium states. However, as we shall see in Sect. 4, Norton makes free use of the idea of a 'non-equilibrium state' without such a defined extension.

\subsection{The Relationship of Demarcation}

Consider the sequence

$$
\mathcal{S}_{m}\left(\mathcal{L}\left(\mathbf{x}_{0}, \mathbf{x}_{1}\right)\right):=\left\{\mathbf{x}\left(\lambda_{i}\right) \mid \mathbf{x}\left(\lambda_{i}\right) \in \mathcal{L}\left(\mathbf{x}_{0}, \mathbf{x}_{1}\right), 0=\lambda_{0}<\lambda_{1}<\cdots \lambda_{m-1}<\lambda_{m}=1\right\}
$$

\footnotetext{
${ }^{9}$ The use of the terms 'reversible' and 'irreversible' italicised by us in this quote should be remarked upon. It is evident from our development that we would prefer, at this stage, that they be respectively replaced by 'equilibrium' and 'non-equilibrium.'

${ }^{10}$ Together, of course, in each case with related parts of speech.
} 


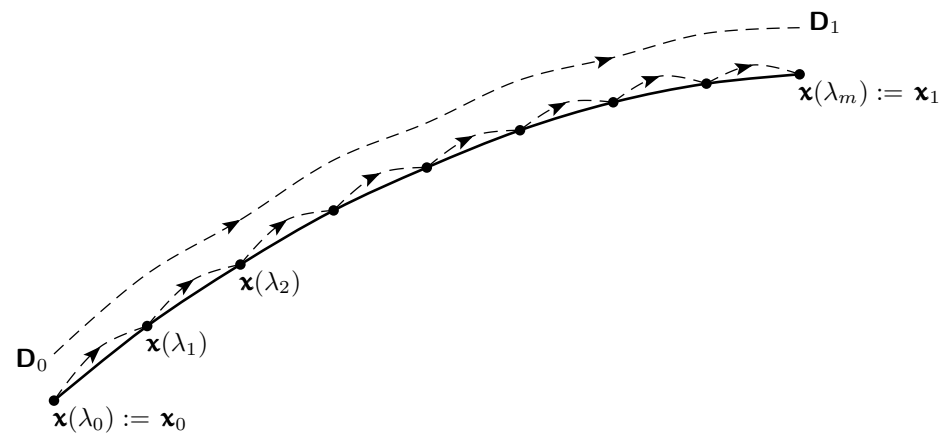

Figure 1: Comparison of delimitation and demarcation. The (continuous) accessibility curve $\mathcal{L}\left(\mathbf{x}_{0}, \mathbf{x}_{1}\right)$ contains the points of a sequence $\mathcal{S}_{m}\left(\mathcal{L}\left(\mathbf{x}_{0}, \mathbf{x}_{1}\right)\right.$ ) (for $m=8$ ). The corresponding sequence of processes demarcated by $\mathcal{L}\left(\mathbf{x}_{0}, \mathbf{x}_{1}\right)$ is shown as broken lines joining the points. The curve from $D_{0}$ to $D_{1}$ is representative of the set of curves which are delimited by $\mathcal{L}\left(\mathbf{x}_{0}, \boldsymbol{x}_{1}\right)$ as proposed by Norton (2016).

of points on the accessible curve $\mathcal{L}\left(\mathbf{x}_{0}, \mathbf{x}_{1}\right)$ and the sequence of processes

$$
\mathbf{x}\left(\lambda_{0}\right) \rightarrow \mathbf{x}\left(\lambda_{1}\right), \quad \mathbf{x}\left(\lambda_{1}\right) \rightarrow \mathbf{x}\left(\lambda_{2}\right), \quad \cdots \quad \mathbf{x}\left(\lambda_{m-1}\right) \rightarrow \mathbf{x}\left(\lambda_{m}\right),
$$

demarcated by $\mathcal{L}\left(\mathbf{x}_{0}, \mathbf{x}_{1}\right)$. An illustrative example of this is given in Fig. 1, where the processes are represented as broken lines, indicative of the fact that they do not trace a path in $\Xi$.

The definition 2-1, given above, is effectively the continuum limit of the sequence of processes (3) as $m \rightarrow \infty$ in such a way that the minimum distance between any two points of $\mathcal{S}_{m}\left(\mathcal{L}\left(\mathbf{x}_{0}, \mathbf{x}_{1}\right)\right)$ tends to zero. The approach to this limit (if it existed) could be construed in two ways: cause and effect.

The cause of a process is the manipulation of the control variables, and approaching the limit would mean that, for each process in the sequence, these manipulations are weakened. The effect is a succession of displacements $\boldsymbol{x}\left(\lambda_{i}\right)$ to $\boldsymbol{x}\left(\lambda_{i+1}\right)$ between equilibrium states on $\mathcal{L}\left(\mathbf{x}_{0}, \mathbf{x}_{1}\right)$. An underlying assumption here is that a slight manipulation of the control variables will lead to only a slight change of equilibrium state. More precisely:

The Hypothesis of Cause and Effect: that in most situations as the manipulations of the control variables are weakened towards zero, the difference between the initial and final state of the process approaches zero.

is adopted. ${ }^{11}$ There is one obvious class of exceptions to this and that arises from the presence of phase transitions, where a small change in an intensive (control) variable will lead, in the case of a first-order transition, to large changes in the extensive (state) variables. For the sake of simplicity such situations will not be considered here, supposing that the whole of the curve $\mathcal{L}\left(\mathbf{x}_{0}, \mathbf{x}_{1}\right)$ lies in the same phase and away from any critical points where, in the case for example of a fluid, critical opalescence entails large inhomogeneities in densities which conflict with a simple idea of the equilibrium state.

The hypotheses of controllability, achievability and cause and effect combine to present a picture where finer and finer sequences $\mathcal{S}_{m}\left(\mathcal{L}\left(\mathbf{x}_{0}, \mathbf{x}_{1}\right)\right)$ of points along $\mathcal{L}\left(\mathbf{x}_{0}, \mathbf{x}_{1}\right)$ are achieved by weakening the manipulations

\footnotetext{
${ }^{11}$ One might be tempted to replace the latter part of this statement by reference to the 'deviation of the state from equilibrium during the process'. However, there is not, without an extension of the theory, a metric for measuring deviations from equilibrium. Whereas a metric for distance between two equilibrium states is easily defined.
} 
of the control variables. However, as has already been made clear, the limit of this succession does not exist. There is no model of thermodynamics which includes the possibility of manipulation of the controls to propel the system along $\mathcal{L}\left(\mathbf{x}_{0}, \mathbf{x}_{1}\right)$. Rather $\mathcal{L}\left(\mathbf{x}_{0}, \mathbf{x}_{1}\right)$ 'demarcates' or is the 'common frontier' of the set of all sequences of processes defined by $\mathcal{S}_{m}\left(\mathcal{L}\left(\mathbf{x}_{0}, \mathbf{x}_{1}\right)\right), \forall m>0$. These can all be regarded as approximations to the 'equilibrium process' defined by $2-1$. But since this process does not exist we are left only with approximations. With this in mind we propose that Defs. 2 are discarded and substituted by:

\section{Replacements for Definitions 2:}

2-1 ${ }^{\star}$ : An equilibrium process along the curve $\mathcal{L}\left(\boldsymbol{x}_{0}, \boldsymbol{x}_{1}\right)$ is the set of all sequences of processes $(3)$ between the points of all $\mathcal{S}_{m}\left(\mathcal{L}\left(\mathbf{x}_{0}, \mathbf{x}_{1}\right)\right), \forall$ integers $m \in(0, \infty)$, demarcated by $\mathcal{L}\left(\mathbf{x}_{0}, \mathbf{x}_{1}\right)$ and resulting from certain allowed manipulations of the control variables. This set of sequences of processes is denoted by $\mathcal{L}\left(\boldsymbol{x}_{0}, \boldsymbol{x}_{1} \mid \mathcal{S}\right)$, where $\mathcal{S}$ is the statement which describes the allowed manipulations of the control variables; that is the type of accessibility.

Of course, given a particular curve $\mathcal{L}\left(\mathbf{x}_{0}, \mathbf{x}_{1}\right), \mathcal{L}\left(\mathbf{x}_{0}, \mathbf{x}_{1} \mid \mathcal{S}\right)$ may not exist in that there are no sequences of processes for that specification of accessibility $\mathcal{S}$.

$\mathbf{2}^{\star} \mathbf{2}^{\star}: \mathcal{L}\left(\mathbf{x}_{0}, \mathbf{x}_{1} \mid \mathcal{S}\right)$ is a reversible equilibrium process if both it and $\mathcal{L}\left(\mathbf{x}_{1}, \mathbf{x}_{0} \mid \mathcal{S}\right)$ exist. If $\mathcal{L}\left(\mathbf{x}_{0}, \mathbf{x}_{1} \mid \mathcal{S}\right)$ exists but not $\mathcal{L}\left(\mathbf{x}_{1}, \mathbf{x}_{0} \mid \mathcal{S}\right)$ it is irreversible. ${ }^{12}$

It should be noted that our notation $\mathcal{L}\left(\mathbf{x}_{0}, \mathbf{x}_{1} \mid \mathcal{S}\right)$ is intended to emphasis the primary role of the curve $\mathcal{L}\left(\mathbf{x}_{0}, \mathbf{x}_{1}\right)$ and the accessibility designation $\mathcal{S}$. Once these are both specified the sequences of processes are secondary in that they are entailed by $\mathcal{L}\left(\mathbf{x}_{0}, \mathbf{x}_{1}\right)$ and $\mathcal{S}$.

It can be seen from the work of Lieb and Yngvason ${ }^{13}$ that a full account of thermodynamics can be given with little reference to curves of states in $\Xi$. The important property is that of accessibility (and more crucially adiabatic accessibility) between pairs of states. The role of accessible curves and sequences of processes demarcated by the curves is largely heuristic. It nevertheless remains to show that they provide an adequate substitute for the usual approach to thermodynamics. But before doing this we need to further distinguish between types of accessibility and reversible and irreversible equilibrium processes. As we have indicated accessibility, in its general unqualified form, has been defined to allow all possible manipulations of the control variables and a usually hidden axiom of thermodynamics is: ${ }^{14}$

The Hypothesis of Accessibility and Recoverability: $\boldsymbol{x} \prec \boldsymbol{x}^{\prime}, \forall \boldsymbol{x}, \boldsymbol{x}^{\prime} \in \Xi$. Meaning, of course, that every state is recoverable from every other state. $\mathcal{L}\left(\mathbf{x}_{0}, \mathbf{x}_{1} \mid \mathcal{S}\right)$ and $\mathcal{L}\left(\mathbf{x}_{1}, \mathbf{x}_{0} \mid \mathcal{S}\right)$ exist, $\forall \mathcal{L}\left(\mathbf{x}_{0}, \mathbf{x}_{1}\right)$ and some S.

The latter part of this statement is our version of Norton's existence assumption (op. cit. p. 45). Of course, not all curves are adiabatically accessible. If they were the second law of thermodynamics would be false.

\subsubsection{Adiabatic Processes}

Adiabatic processes and adiabatic accessibility are a consequence of restrictions placed on the controlvariable manipulations which can be involved in implementing the process. ${ }^{15}$ The assertion that "an adia-

\footnotetext{
${ }^{12}$ It is a moot point whether we use the terms 'reversible' and 'irreversible' or 'recoverable' and irrecoverable' here. However, since we would argue that the former terms have no role other than that given to them here, and that they reflect the reverse parametrization of the curve, we propose to adopt this terminology.

${ }^{13}$ The most comprehensive account is given in Lieb and Yngvason (1999), with briefer versions in Lieb and Yngvason (1998) and Lieb and Yngvason (2000).

${ }^{14}$ The use of the accessibility condition $\mathcal{S}$ in this hypothesis needs a slight gloss in two respects. Firstly, as indicated above, the curve $\mathcal{L}\left(\mathbf{x}_{0}, \mathbf{x}_{1}\right)$ may be piecewise divisible into parts with different manipulations of different control variables needed to achieve accessibility. Secondly, different manipulations of different control variable may be needed for accessibility for $\mathcal{L}\left(\mathbf{x}_{0}, \mathbf{x}_{1}\right)$ and $\mathcal{L}\left(\boldsymbol{x}_{1}, \boldsymbol{x}_{0}\right)$. $\mathcal{S}$ is the union of all these different types of manipulation.

${ }^{15}$ As already noted, Lieb and Yngvason use $\prec$ to denote adiabatic accessibility, while we use $\prec$ to denote this more restricted

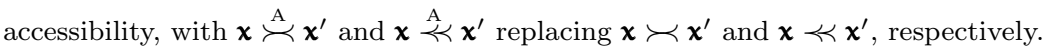


batic process is characterized physically by the absence of any thermal interaction between the system and its environment" (Boyling, 1972, p. 36) leaves some uncertainty about what counts as a thermal interaction, or more specifically what is allowed as a 'non-thermal' interaction. According to Lieb and Yngvason (1999, p. 17$):{ }^{16}$

A state $\boldsymbol{x}^{\prime}$ is adiabatically accessible from a state $\boldsymbol{x}$ if it is possible to change the state $\boldsymbol{x}$ to $\boldsymbol{x}^{\prime}$ by means of an interaction with some device (which may consist of mechanical and electrical parts as well as auxiliary thermodynamic systems) and a weight, in such a way that the device returns to its initial state at the end of the process whereas the weight may have changed its position in a gravitational field. Thus the only work done is by/to the weight and the loss/gain in its potential energy will be equal to the gain/loss of internal energy in the system.

The point about an adiabatic process, as stated in the first law, is contained in the last sentence in this quote; the work done on/by the system by/on its environment is equal to the increase/decrease in internal energy. Lieb and Yngvason make this specific by following Joule (1850), who used a weight falling under gravity as a measure of the work done on a system in his discussions of the mechanical equivalence of heat. Once this is done all manner of ingenuity can be used to link the falling weight to the system, leading to a variety of possible changes to the state of the system, predicated only on the linking mechanisms returning to their initial states. An obvious example is a set of levers causing the expansion or compression of a fluid. Here there is a change in a deformation variable. However, a too close association between adiabatic processes and changes in the deformation variables would be a mistake. A falling weight can be used to cause rubbing, stirring or electrical heating of a system without any change in the deformation variables. We should, however, note that the comment by Lieb and Yngvason (op. cit. p. 18) that:

In the usual parlance, rubbing would be an adiabatic process, but not electrical 'heating', because the latter requires the introduction of a pair of wires through the 'adiabatic enclosure'.

is at variance with Buchdahl (1966, Sect. 1.8) who asserts that:

[A system in an adiabatic enclosure] may incorporate a stirrer, or an electrical resistance through which a current may be passed from outside the enclosure, and so on. Then the passage of such a current, movement of the stirrer, variation of the deformation coordinates, are all to be counted as mechanical processes.

There is, however, a novel element in Lieb and Yngvason's definition and that is the inclusion of an "auxiliary thermodynamic system". This lies at the heart of the proof that all Cartesian products like $\Xi_{1} \times \Xi_{2}$ of simple systems $\Xi_{1}$ and $\Xi_{2}$ are comparable. That is to say, for all pairs of states $\boldsymbol{x}_{1}, \boldsymbol{x}_{1}^{\prime} \in \Xi_{1}$ and $\boldsymbol{x}_{2}, \boldsymbol{x}_{2}^{\prime} \in \Xi_{2}$ either $\left(\mathbf{x}_{1}, \mathbf{x}_{2}\right) \stackrel{\mathrm{A}}{\prec}\left(\mathbf{x}_{1}^{\prime}, \mathbf{x}_{2}^{\prime}\right)$ or $\left(\mathbf{x}_{1}^{\prime}, \mathbf{x}_{2}^{\prime}\right) \stackrel{\mathrm{A}}{\prec}\left(\mathbf{x}_{1}, \mathbf{x}_{2}\right)$.

An illuminating element of Lieb and Yngvason's account of thermodynamics is the use of forward sectors Lieb and Yngvason (op. cit. Sects. 2.6 and 3.3). The set

$$
\mathcal{F}_{\mathbf{x}}:=\left\{\boldsymbol{x}^{\prime} \in \Xi \mid \mathbf{x} \stackrel{\mathrm{A}}{\prec} \boldsymbol{x}^{\prime}\right\}
$$

is the forward sector of $\mathbf{x}$ which is shown to have the properties that:

(a) $\mathcal{F}_{\boldsymbol{X}}$ is a relatively closed convex subset of $\Xi .^{17}$

(b) The boundary $\partial \mathcal{F}_{\mathbf{x}} \in \Xi$ of $\mathcal{F}_{\boldsymbol{x}}$ contains $\boldsymbol{x}$ and:

(i) $\boldsymbol{x}^{\prime} \stackrel{\mathrm{A}}{\prec} \boldsymbol{x} \Longleftrightarrow \boldsymbol{x}^{\prime} \in \partial \mathcal{F}_{\boldsymbol{x}}$; that is $\partial \mathcal{F}_{\boldsymbol{x}}$ is an adiabatic surface.

(ii) $\boldsymbol{x} \stackrel{\mathrm{A}}{\gtrless} \boldsymbol{x}^{\prime} \Longleftrightarrow \boldsymbol{x}^{\prime} \in \mathcal{F}_{\mathbf{x}}^{\circ}$, the interior of $\mathcal{F}_{\mathbf{x}}$.

(c) Forward sectors are nested with:

\footnotetext{
${ }^{16}$ We have adapted slightly to our notation.

${ }^{17}$ Relative closure is needed since $\mathcal{F}_{\mathbf{x}}$ is bounded in part by the boundary of $\Xi$ which is an open set.
} 
(i) $\boldsymbol{x}^{\prime} \stackrel{\mathrm{A}}{\prec} \boldsymbol{x} \Longleftrightarrow \mathcal{F}_{\boldsymbol{x}}=\mathcal{F}_{\boldsymbol{x}^{\prime}}$.

(ii) $\boldsymbol{x} \stackrel{\text { A }}{\gtrless} \boldsymbol{x}^{\prime} \Longleftrightarrow \mathcal{F}_{\mathbf{x}^{\prime}} \subset \mathcal{F}_{\mathbf{x}}^{\circ}$.

(d) For positive absolute temperature ${ }^{18} \partial \mathcal{F}_{\mathbf{x}}$ is concave upwards with respect to the direction of the thermal axis.

An interesting difference between the approach of Lieb and Yngvason and accounts of thermodynamics based on the statement of the second law by Carathéodory $(1909)^{19}$ is that in the latter case (see, e.g. Buchdahl, 1966, Chap. 5) the existence of entropy and temperature are derived as consequences of the second law, whereas in the former, the existence of an entropy function $S(\mathbf{x})$ and temperature $T(\mathbf{x})$, with entropy having the appropriate additive and scaling properties and such that

$$
\begin{array}{ll}
\boldsymbol{x} \stackrel{\mathrm{A}}{\gtrless} \mathbf{x}^{\prime} & \Longleftrightarrow \quad S(\mathbf{x})<S\left(\mathbf{x}^{\prime}\right), \\
\mathbf{x} \stackrel{\mathrm{A}}{\gtrless} \mathbf{x}^{\prime} \quad \Longleftrightarrow \quad S(\mathbf{x})=S\left(\mathbf{x}^{\prime}\right),
\end{array}
$$

and Carathéodory's form of the second law are all consequences of the axiomatic structure.

It follows, from Def. 1 that $\mathcal{L}\left(\mathbf{x}_{0}, \mathbf{x}_{1}\right)$ is, $\forall i<j$ :

(i) Adiabatically accessible if $\mathbf{x}\left(\lambda_{j}\right) \in \mathcal{F}_{\mathbf{x}\left(\lambda_{i}\right)}$ or equivalently that $S\left(\mathbf{x}\left(\lambda_{i}\right)\right) \leq S\left(\mathbf{x}\left(\lambda_{j}\right)\right)$,

(ii) Adiabatically reversible if $\mathbf{x}\left(\lambda_{j}\right) \in \partial \mathcal{F}_{\mathbf{x}\left(\lambda_{i}\right)}$ or equivalently that $S\left(\mathbf{x}\left(\lambda_{i}\right)\right)=S\left(\mathbf{x}\left(\lambda_{j}\right)\right)$,

(iii) Adiabatically irreversible if $\mathbf{x}\left(\lambda_{j}\right) \in \mathcal{F}_{\mathbf{x}\left(\lambda_{i}\right)}^{\circ}$ or equivalently that $S\left(\mathbf{x}\left(\lambda_{i}\right)\right)<S\left(\mathbf{x}\left(\lambda_{j}\right)\right)$.

An adiabatic equilibrium process $\mathcal{L}\left(\mathbf{x}_{0}, \mathbf{x}_{1} \mid \mathcal{A}\right)$ is defined as in $2-1^{\star}$, with $\mathcal{A}$ describing the manipulations allowed for adiabatic processes. Reversible and irreversible adiabatic equilibrium process are similarly defined like $2-2^{\star}$.

It follows that, for $\mathcal{L}\left(\mathbf{x}_{0}, \mathbf{x}_{1} \mid \mathcal{A}\right)$ :

(a) Entropy increases from $\boldsymbol{x}_{0}$ to $\boldsymbol{x}_{1}$.

(b) Entropy strictly increases from $\boldsymbol{x}_{0}$ to $\boldsymbol{x}_{1}$ if $\mathcal{L}\left(\mathbf{x}_{0}, \mathbf{x}_{1} \mid \mathcal{A}\right)$ is irreversible, and this is the case iff, for all sequences $(2), \mathcal{F}_{\mathbf{x}\left(\lambda_{j}\right)} \subset \mathcal{F}_{\mathbf{x}\left(\lambda_{i}\right)}^{\circ}, \forall i<j$

(c) Entropy remains constant if $\mathcal{L}\left(\mathbf{x}_{0}, \mathbf{x}_{1} \mid \mathcal{A}\right)$ is reversible, and this is the case iff $\mathcal{L}\left(\mathbf{x}_{0}, \mathbf{x}_{1}\right) \subset \partial \mathcal{F}_{\mathbf{x}_{0}}$.

\subsubsection{Isothermal Processes}

A process $\mathbf{x} \rightarrow \mathbf{x}^{\prime}$ is isothermal iff the temperature is the same at the beginning and end of the process; that is $T(\boldsymbol{x})=T\left(\boldsymbol{x}^{\prime}\right)$. It follows from the hypothesis of accessibility and recoverability that there is a recoverable isothermal process between a pair of points $\boldsymbol{x}, \boldsymbol{x}^{\prime} \in \Xi$ iff $T(\boldsymbol{x})=T\left(\boldsymbol{x}^{\prime}\right) . \mathcal{L}\left(\boldsymbol{x}_{0}, \mathbf{x}_{1}\right)$ is an isotherm if $T(\boldsymbol{x}(\lambda))=T\left(\mathbf{x}_{0}\right), \forall \lambda \in[0,1]$ and then the processes of any sequence demarcated by the curve and in either direction are isothermal. Denoting by $\mathcal{J}_{T}$ the manipulations needed to effect processes between points with equal temperature $T$, the isothermal equilibrium process $\mathcal{L}\left(\mathbf{x}_{0}, \mathbf{x}_{1} \mid \mathcal{J}_{T}\right)$ is defined as in $2-1^{\star}$. It exists iff $\mathcal{L}\left(\mathbf{x}_{0}, \mathbf{x}_{1}\right)$ is an isotherm at temperature $T$ and then it is always reversible.

Isotherms lie on isothermal surfaces in $\Xi .^{20}$ Remembering that the one thermal variable $\chi^{\mathrm{T}}$ of $\Xi$ is the internal energy $U$, the temperature is given by

$$
T(\boldsymbol{x})=\left(\frac{\partial S\left(\chi^{\mathrm{T}}, \boldsymbol{x}^{\mathrm{D}}\right)}{\partial \chi^{\mathrm{T}}}\right)^{-1}
$$

A standard method to implement the manipulations described by $\mathcal{J}_{T}$ is to use an isothermal reservoir $\Xi_{\mathrm{R}}$. This is a simple system whose temperature $T_{\mathrm{R}}$ remains constant, by virtue of its entropy being a

\footnotetext{
${ }^{18}$ The case of negative absolute temperature is of interest, but will not be considered here.

${ }^{19}$ This appears as his Axiom II on page 236.

${ }^{20}$ Although, certain properties of continuity and smoothness, with which adiabatic surfaces are endowed in the account of Lieb and Yngvason, are not necessarily shared by isothermal surfaces.
} 
linear function of its thermal variable $x_{R}^{T}$, and such that, in any process its deformation variable $\boldsymbol{x}_{R}^{\mathrm{D}}$ also remains constant. This means that the only processes allowed are demarcated by the thermal $x_{\mathrm{R}}^{\mathrm{T}}$-axis with $T\left(\mathbf{x}_{\mathrm{R}}\right):=T_{\mathrm{R}}$.

Given that $\Xi$ is in state $\boldsymbol{x}_{0}$ with $T\left(\mathbf{x}_{0}\right)=T_{\mathrm{R}}, \Xi$ and $\Xi_{\mathrm{R}}$, in state $\boldsymbol{x}_{\mathrm{R} 0}$, can be placed in thermal contact to form the joined system $\bar{\Xi}$ in state $\overline{\mathbf{x}}:=\left(\bar{x}_{0}^{\mathrm{T}}, \mathbf{x}_{0}^{\mathrm{D}}, \mathbf{x}_{\mathrm{R}}^{\mathrm{D}}\right)$, where $\overline{\boldsymbol{x}}_{0}^{\mathrm{T}}=x_{0}^{\mathrm{T}}+\boldsymbol{x}_{\mathrm{R} 0}^{\mathrm{T}}$, without any flow of internal energy in terms of heat between $\Xi$ and $\Xi_{\mathrm{R}} \cdot{ }^{21}$ Consider now the adiabatic accessible curve $\overline{\mathcal{L}}\left(\overline{\mathbf{x}}_{0}, \overline{\mathbf{x}}_{1}\right)$ in $\Xi$ and an adiabatic process $\overline{\boldsymbol{x}}\left(\lambda_{i}\right) \rightarrow \overline{\mathbf{x}}\left(\lambda_{i+1}\right)$ in a sequence like (2) demarcated by $\overline{\mathcal{L}}\left(\overline{\boldsymbol{x}}_{0}, \overline{\boldsymbol{x}}_{1}\right)$. If $\bar{\Xi}$ were split and rejoined at the end of each process in the sequence this would correspond to processes $\mathbf{x}\left(\lambda_{i}\right) \rightarrow \mathbf{x}\left(\lambda_{i+1}\right)$ and $\boldsymbol{x}_{\mathrm{R}}\left(\lambda_{i}\right) \rightarrow \boldsymbol{x}_{\mathrm{R}}\left(\lambda_{i+1}\right)$ demarcated by isothermal curves $\mathcal{L}\left(\mathbf{x}_{0}, \boldsymbol{x}_{1}\right)$ and $\mathcal{L}_{\mathrm{R}}\left(\mathbf{x}_{\mathrm{R} 0}, \boldsymbol{x}_{\mathrm{R} 1}\right)$ in $\Xi$ and $\Xi_{\mathrm{R}}$ respectively. The set of all sequences of this type for $\Xi$ is simply the isothermal equilibrium process $\mathcal{L}\left(\mathbf{x}_{0}, \mathbf{x}_{1} \mid \mathcal{J}_{T_{\mathrm{R}}}\right)$.

According to the first law of thermodynamics, for adiabatic accessibility, if $\overline{\mathbf{x}} \stackrel{\mathrm{A}}{\prec} \overline{\boldsymbol{x}}^{\prime}$ there exists an adiabatic work function $W\left(\overline{\mathbf{x}}, \overline{\mathbf{x}}^{\prime}\right)$, which satisfies appropriate transitivity, additivity and scaling properties. It can also be shown (Boyling, 1972, p. 38) that $W\left(\overline{\mathbf{x}}, \overline{\mathbf{x}}^{\prime}\right)=\bar{\chi}^{\mathrm{T}}-\overline{\boldsymbol{x}}^{\mathrm{T}^{\prime}} \cdot{ }^{22}$ Then

$$
W_{i}:=W\left(\overline{\mathbf{x}}\left(\lambda_{i}\right), \overline{\mathbf{x}}\left(\lambda_{i+1}\right)\right)=-\triangle_{i} \chi_{\mathrm{R}}^{\mathrm{T}}-\triangle_{i} \chi^{\mathrm{T}}
$$

is the work done by $\bar{\Xi}$ on the environment during the adiabatic process $\overline{\mathbf{x}}\left(\lambda_{i}\right) \rightarrow \overline{\mathbf{x}}\left(\lambda_{i+1}\right)$, where

$$
\triangle_{i} \chi_{\mathrm{R}}^{\mathrm{T}}:=\chi_{\mathrm{R}}^{\mathrm{T}}\left(\lambda_{i+1}\right)-\chi_{\mathrm{R}}^{\mathrm{T}}\left(\lambda_{i}\right), \quad \triangle_{i} \chi^{\mathrm{T}}:=\chi^{\mathrm{T}}\left(\lambda_{i+1}\right)-\chi^{\mathrm{T}}\left(\lambda_{i}\right)
$$

Given that heat $Q_{i}$ flows from $\Xi_{\mathrm{R}}$ into $\Xi$ and work on the environment is performed entirely by $\Xi$,

$$
\begin{aligned}
& \triangle_{i} \mathrm{x}_{\mathrm{R}}^{\mathrm{T}}=-Q_{i}, \quad \triangle_{i} \mathrm{x}^{\mathrm{T}}=-W_{i}+Q_{i}, \\
& \frac{Q_{i}}{T_{\mathrm{R}}}=-\triangle_{i} S_{\mathrm{R}} \leq \triangle_{i} S, \\
& \triangle_{i} S_{\mathrm{R}}:=S_{\mathrm{R}}\left(\mathbf{x}_{\mathrm{R}}\left(\lambda_{i+1}\right)\right)-S_{\mathrm{R}}\left(\mathbf{x}_{\mathrm{R}}\left(\lambda_{i}\right)\right), \quad \triangle_{i} S:=S\left(\mathbf{x}\left(\lambda_{i+1}\right)\right)-S\left(\boldsymbol{x}\left(\lambda_{i}\right)\right) .
\end{aligned}
$$

As we have noted in point (v) in Sect. 1, $\boldsymbol{x}\left(\lambda_{i}\right) \rightarrow \mathbf{x}\left(\lambda_{i+1}\right)$ does not represent a unique specification for the way that the state $\boldsymbol{x}\left(\lambda_{i}\right)$ of $\Xi$ is changed to $\boldsymbol{x}\left(\lambda_{i+1}\right)$. With fixed $\triangle_{i} \chi^{\mathrm{T}}$ and $\triangle_{i} S$ these different possibilities correspond to different inputs $Q_{i}$ and $W_{i}$, and consequentially different resulting states $\boldsymbol{x}_{\mathrm{R}}\left(\lambda_{i+1}\right)$ for the reservoir such that $Q_{i} \leq T_{\mathrm{R}} \triangle_{i} S$ and $W_{i}=Q_{i}-\triangle_{i} \chi^{\mathrm{T}}$. Although a strict inequality in (10) implies that $\overline{\mathbf{x}}\left(\lambda_{i}\right) \stackrel{\text { }}{\gtrless} \overline{\mathbf{x}}\left(\lambda_{i+1}\right)$ this does not imply (non-adiabatic) irrecoverability in $\Xi$. For $\mathbf{x}\left(\lambda_{i+1}\right) \rightarrow \mathbf{x}\left(\lambda_{i}\right)$ one simply needs inputs $Q_{i}^{\prime}$ and $W_{i}^{\prime}$ satisfying $Q_{i}^{\prime} \leq-T_{\mathrm{R}} \triangle_{i} S$ and $W_{i}^{\prime}=Q_{i}^{\prime}+\triangle_{i} \chi^{\mathrm{T}}$ giving $\boldsymbol{x}_{\mathrm{R}}\left(\lambda_{i+1}\right) \rightarrow \boldsymbol{x}_{\mathrm{R}}^{\prime}\left(\lambda_{i}\right)$; meaning that the final state of the reservoir for this process is in general different from the initial state $\boldsymbol{x}_{\mathrm{R}}\left(\lambda_{i}\right)$.

That is, except in the special case where $\overline{\mathbf{x}}\left(\lambda_{i}\right) \stackrel{\mathrm{A}}{\prec} \overline{\mathbf{x}}\left(\lambda_{i+1}\right)$, with equality in (10), when there are processes $\boldsymbol{x}\left(\lambda_{i}\right) \rightarrow \boldsymbol{x}\left(\lambda_{i+1}\right)$ and $\mathbf{x}\left(\lambda_{i+1}\right) \rightarrow \boldsymbol{x}\left(\lambda_{i}\right)$ with $Q_{i}^{\prime}=-Q_{i}$ and $W_{i}^{\prime}=-W_{i}$.

The relationships (9)-(11) apply to sets of sequences (3) of processes $\boldsymbol{x}\left(\lambda_{i}\right) \rightarrow \boldsymbol{x}\left(\lambda_{i+1}\right)$ for $\Xi$ and thus to the equilibrium isothermal process $\mathcal{L}\left(\mathbf{x}_{0}, \mathbf{x}_{1} \mid \mathcal{J}_{T_{\mathrm{R}}}\right)$.

\section{Examples}

We now consider examples of accessible curves in a perfect fluid and of those forming a cycle between two reservoirs. As indicated above, we shall take these curves as demarcating sequences of processes and thus, together with a description of the accessibility, as defining equilibrium processes. Norton also uses these examples to illustrate equilibrium processes. The difference, as we shall explore it in Sect. 4 , is in the relationship between the sets of processes and the curve for which he uses the term delimiting and the sets of sequences of processes and the curve for which we use the term demarcating. In most of this section the terms 'demarcating' or 'delimiting' can equally well be used since the exact relationship between the curve and the processes is not critical.

\footnotetext{
${ }^{21}$ That systems can be thermally joined and subsequently split as described here, follows from the axioms of Lieb and Yngvason. In most presentations of thermodynamics it tends to be regarded as self-evident.

${ }^{22}$ In fact the proof demands that $\Xi$ is an adiabatically directed set, a condition which Boyling incorporates into his statement of the first law. That the increase in internal energy of the system is equal to minus the work done during the process is just due to a convenient choice of sign.
} 


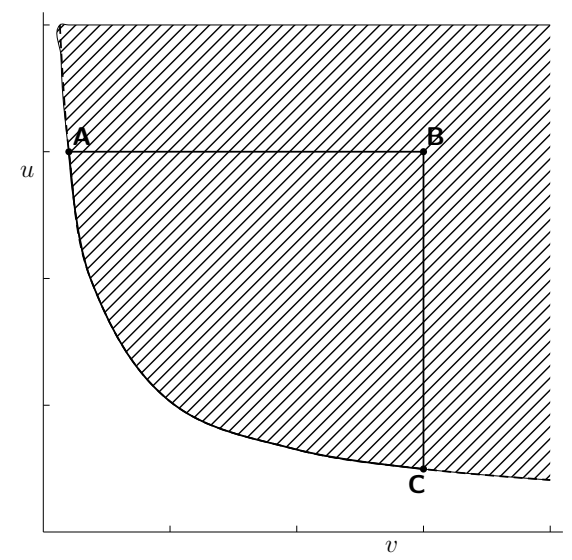

Figure 2: The space $\Xi$ for the perfect monatomic fluid. A, B and C label the states $\boldsymbol{x}_{\mathrm{A}}, \boldsymbol{x}_{\mathrm{B}}$ and $\boldsymbol{x}_{\mathrm{C}}$. The forward sector of the adiabat through $A$ and $C$ is shaded.

\subsection{The Perfect Monatomic Fluid}

For a perfect fluid of $N$ monatomic particles of mass $m, \Xi$ can be taken to be the space of $\mathbf{x}:=(u, v)$, where $u$ and $v$ are respectively the internal energy and volume per particle. The entropy per particle is ${ }^{23}$

$$
s(u, v):=c+\frac{3}{2} \ln (u)+\ln (v), \quad \text { where } \quad c:=\frac{5}{2}+\frac{3}{2} \ln \left(\frac{4 \pi m}{3 h^{2}}\right),
$$

and $h$ is Planck's constant. The temperature and pressure, given respectively as

$$
T(u, v)=\left(\frac{\partial s}{\partial u}\right)^{-1}=\frac{2}{3} u, \quad P(u, v)=T(u, v) \frac{\partial s}{\partial v}=\frac{T(u, v)}{v}=\frac{2 u}{3 v},
$$

are the control variables, with the effect of the pressure produced by the force exerted on a piston closing the cylinder containing the fluid, and the temperature mediating the internal energy through equilibrium contact with an isothermal reservoir, as described in Sect. 2.1.2. In the space $\Xi$ given in Fig. 2, the forward sector of $\boldsymbol{x}_{\mathrm{A}}$ (and $\boldsymbol{x}_{\mathrm{C}}$ ) is shaded and bounded by the adiabat through these two states. Thus the adiabatic equilibrium process $\mathcal{L}\left(\mathbf{x}_{\mathrm{A}}, \mathbf{x}_{\mathrm{C}} \mid \mathcal{A}\right)$ is reversible and $\mathcal{L}\left(\mathbf{x}_{\mathrm{A}}, \boldsymbol{x}_{\mathrm{B}} \mid \mathcal{A}\right)$ and $\mathcal{L}\left(\boldsymbol{x}_{\mathrm{C}}, \boldsymbol{x}_{\mathrm{B}} \mid \mathcal{A}\right)$ are irreversible. In addition because of the special relationship, given in (13), between the temperature and internal energy in a perfect fluid $T\left(\mathbf{x}_{\mathrm{A}}\right)=T\left(\mathbf{x}_{\mathrm{B}}\right) ; \mathcal{L}\left(\mathbf{x}_{\mathrm{A}}, \boldsymbol{x}_{\mathrm{B}}\right)$ is an isotherm and the (reversible) isothermal equilibrium process $\mathcal{L}\left(\mathbf{x}_{\mathrm{A}}, \boldsymbol{x}_{\mathrm{B}} \mid J_{T_{\mathrm{A}}}\right)$ exists. Then:

- Adiabatically irrecoverable processes delimited/demarcated by $\mathcal{L}\left(\boldsymbol{x}_{\mathrm{\subset}}, \boldsymbol{x}_{\mathrm{B}}\right)$ can, as described in Sect. 2.1.1, be implemented by introducing an electrical heating element into the fluid and powering the current by the falling of a weight.

- Norton (op. cit. p. 57) proposes two possible ways to implement a sequence of adiabatically irrecoverable processes delimited (in his case, but equally well demarcated in our case) by $\mathcal{L}\left(\mathbf{x}_{\mathrm{A}}, \mathbf{x}_{\mathrm{B}}\right)$. In the first the cylinder is thermally isolated and a sequence of closely-spaced membranes contain the fluid in a part of the cylinder with volumes $v_{\mathrm{A}}=: v\left(\lambda_{0}\right)<v\left(\lambda_{1}\right)<\cdots<v\left(\lambda_{m}\right):=v_{\mathrm{B}}$. The membranes are successively broken with the fluid expanding without work being expended or the internal energy changing. Alternatively the piston closing the cylinder could be such that its motion is so impeded by friction that it barely moves. In a succession of small expansions the work expended on it by the fluid is returned to the fluid as heat generated by the friction.

\footnotetext{
${ }^{23}$ Variables are given here in the field-density representation of Lavis (2015) in which entropy and temperature absorb Boltzmann's constant to become, respectively, dimensionless and of the dimensions of energy.
} 


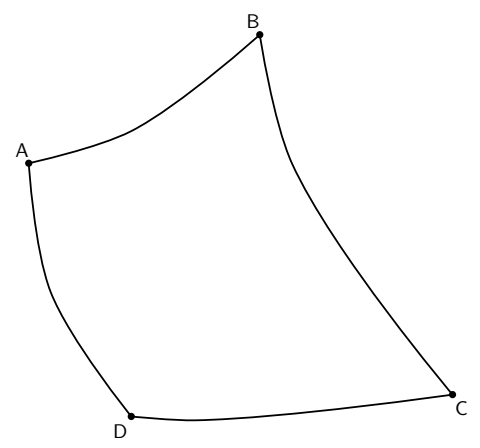

Figure 3: A cycle of states. A, B, C and D label the states $\boldsymbol{x}_{A}, \boldsymbol{x}_{B}, \boldsymbol{x}_{C}$ and $\boldsymbol{x}_{D}$. AB and CD are isotherms and BC and DA are adiabats.

- From (12) the adiabat $\mathcal{L}\left(\mathbf{x}_{\mathrm{A}}, \mathbf{x}_{\mathrm{C}}\right)$ has $u^{3} v^{2}$ equal to some constant value. This curve delimits/demarcates a sequence of processes where the fluid in the cylinder is thermally isolated and the piston reduces or increases the volume in small steps. For each process the work done on or by the piston is equal to the change in internal energy.

\subsection{A Cycle of Processes}

We now consider the classic case of four states $\boldsymbol{x}_{A}, \boldsymbol{x}_{B}, \boldsymbol{x}_{c}, \mathbf{x}_{D} \in \Xi$, with $B C$ and DA being adiabats linked by isotherms $\mathrm{AB}$ and $\mathrm{CD}$ at temperatures $T_{\mathrm{AB}}>T_{\mathrm{CD}}$. As in our previous discussion the adiabats and isotherms delimit/demarcate sets-of/sets-of-sequences-of processes. In the case of demarcation (but not delimitation) relationships obtained for the individual processes of the sequence can be summed to yield relationships applying to the whole curves. In this respect it should be noted that Lieb and Yngvason (op. cit. p. 73) derive the same results, simply considering the processes between the four points of the cycle without any mention of the curves which can connect them.

The isothermal processes are effected by thermally joining $\Xi$ to isothermal reservoirs and, from (8)-(11), the changes in internal energy and entropy and the work performed on the environment and heat absorbed by the system are given (with obvious notation) by

$$
\begin{array}{ll}
x_{\mathrm{B}}^{\mathrm{T}}-x_{\mathrm{A}}^{\mathrm{T}}=Q_{\mathrm{AB}}-W_{\mathrm{AB}}, & \frac{Q_{\mathrm{AB}}}{T_{\mathrm{AB}}} \leq S_{\mathrm{BC}}-S_{\mathrm{DA}}, \\
x_{\mathrm{D}}^{\mathrm{T}}-x_{\mathrm{C}}^{\mathrm{T}}=Q_{\mathrm{CD}}-W_{\mathrm{CD}}, & \frac{Q_{\mathrm{CD}}}{T_{\mathrm{CD}}} \leq S_{\mathrm{DA}}-S_{\mathrm{BC}} .
\end{array}
$$

for processes $\boldsymbol{x}_{\mathrm{A}} \rightarrow \mathbf{x}_{\mathrm{B}}$ and $\mathbf{x}_{\mathrm{C}} \rightarrow \mathbf{x}_{\mathrm{D}}$, respectively. For adiabatic processes $\mathbf{x}_{\mathrm{B}} \rightarrow \mathbf{x}_{\mathrm{C}}$ and $\boldsymbol{x}_{\mathrm{D}} \rightarrow \boldsymbol{x}_{\mathrm{A}}$,

$$
x_{\mathrm{C}}^{\mathrm{T}}-x_{\mathrm{B}}^{\mathrm{T}}=-W_{\mathrm{BC}}, \quad x_{\mathrm{A}}^{\mathrm{T}}-x_{\mathrm{D}}^{\mathrm{T}}=-W_{\mathrm{DA}} .
$$

Regarding the system as a heat engine, when heat $Q_{\mathrm{AB}}>0$ is extracted from the hot reservoir and total work $W_{\mathrm{T}}=W_{\mathrm{AB}}+W_{\mathrm{BC}}+W_{\mathrm{CD}}+W_{\mathrm{DA}}$ performed on the environment, the heat $-Q_{\mathrm{CD}}>0$ input into the cold reservoir is regarded as wasted. The efficiency of the heat engine

$$
\eta_{\mathrm{HE}}:=1-\frac{\left(-Q_{\mathrm{CD}}\right)}{Q_{\mathrm{AB}}} \in[0,1]
$$

increases as the quantity of wasted heat decreases, with from (14) and (15),

$$
\eta_{\mathrm{HE}} \leq 1-\frac{T_{\mathrm{CD}}}{T_{\mathrm{AB}}}=: \eta_{\mathrm{C}}<1 .
$$

The cycle becomes a Carnot cycle with Carnot efficiency $\eta_{\mathrm{C}}$ when the inequalities in (14) become equalities. Then there is no increase in entropy in the adiabatic processes for the system thermally joined to the 
reservoirs and the cycle is reversible. That the efficiency is bounded above by the Carnot efficiency so that the wasted heat cannot be reduced to zero is equivalent to the Kelvin-Planck version of the second law for positive temperatures. $^{24}$

\section{Delimitation or Demarcation}

Given the curve $\mathcal{L}\left(\mathbf{x}_{0}, \mathbf{x}_{1}\right)$ of equilibrium states in the thermodynamic space $\Xi$, the aim, both of the present work and that of Norton (2016), is to present a credible alternative to the incredible idea that processes exist in which the state of the system changes in a continuous manner along $\mathcal{L}\left(\mathbf{x}_{0}, \mathbf{x}_{1}\right)$. In each approach sets of processes are in some way related to $\mathcal{L}\left(\mathbf{x}_{0}, \mathbf{x}_{1}\right)$. We have found it convenient to coin a distinction between the terms delimitation and demarcation to describe the accounts of this relationship in Norton's and our work, respectively. To explore in detail the difference between these two approaches it is necessary first to review the fundamental perception of the nature of thermodynamics adopted in this paper with a view to exposing the different underlying account implicit in the notion of delimitation.

In Sect. 1 we asserted that the states of thermodynamics are equilibrium states identified with points in a space $\Xi$ of extensive variables. Implicitly understood here is that equilibrium states are states attainable by the system and explicitly stated is the assertion that the system will leave its equilibrium state only by the agency of the control variables, with all the interactions between the system and its environment being of this kind (the hypothesis of controllability). A process is then defined as a manipulation of the control variables to change the state of the system, with all possible processes being achievable exactly and in a finite amount of time (the hypothesis of achievability). That this is essentially the basis for 'ordinary' thermodynamics can be seen from almost any standard account. Thus:

(i) "Given any physical system [...] a finite set of finite macroscopic variables [...] can be listed, such that knowledge of a set of values of these variables defines one of a known sequence of equilibrium states of the system," (Landsberg, 1956, p. 365).

(ii) "The equilibrium of a thermodynamic system is characterized uniquely by the values of a finite set of physical quantities," (Buchdahl, 1966, p. 9) and "systems which cannot attain equilibrium under appropriate experimental conditions will henceforth be excluded from consideration,." (ibid, p. 8).

(iii) "Operationally, a system is in an equilibrium state if its properties are consistently described by thermodynamic theory," 25 (Callen, 1985, p. 15) and "a real process always involves non-equilibrium intermediate states having no representation in the thermodynamic configuration space," (ibid, p. 96).

That this picture constitutes ordinary thermodynamics is effectively acknowledged by Norton (op. cit. p. 50 , our italics) when he writes that his account of equilibrium processes (as we describe it below): ${ }^{26}$

[...] includes an existence assumption. ${ }^{27}$ Whether it obtains depends on the physics governing dynamical processes. It most likely does not fail as long as we neglect the molecular constitution of matter. More precisely, it will hold most likely in a fictitious world in which ordinary thermodynamics holds exactly at all scales.

In Norton's delimiting relationship the sequence of processes (3), which in our scheme is demarcated by $\mathcal{L}\left(\mathbf{x}_{0}, \mathbf{x}_{1}\right)$, is replaced by a single process passing through a succession of non-equilibrium states. Such a process is shown by the curve between $D_{0}$ and $D_{1}$ in Fig. 1; this being represented by a broken line to indicate that none of its points (including its end points) lie in the space $\Xi$ of equilibrium states. Then the two pictures are:

- In our case, as specified in Def. $2-1^{\star}$, the set of all sequences of processes demarcated by $\mathcal{L}\left(\mathbf{x}_{0}, \mathbf{x}_{1}\right)$.

\footnotetext{
${ }^{24}$ This version of the second law is violated for negative temperatures.

${ }^{25}$ In the text this quote is italicised and terminated by an explanation mark to highlight the circular nature of this sentence as a definition of equilibrium. However, it is observed that this circularity is "not fundamentally different from that of mechanics."

${ }^{26}$ Again, as we have already observed, his preferred term is 'reversible processes'.

${ }^{27}$ This, as indicated above, is effectively equivalent to our hypothesis of existence and recoverability.
} 
- In Norton's case the set of non-equilibrium processes approximating ever more closely to the delimiting curve $\mathcal{L}\left(\mathbf{x}_{0}, \mathbf{x}_{1}\right)$.

Common ground is that there is no limit which can be called an equilibrium process and which corresponds to passage along the curve itself. Instead the set of sequences-of-processes/non-equilibrium-processes is itself taken to be the equilibrium process (our Def. 2.1* and Norton's definition at the beginning of his Sect. 2.2). However, Norton (op. cit. p. 45) argues that "while they may come arbitrarily close, none of the states of the irreversible processes are exactly equilibrium states. For otherwise the processes cannot complete in any finite time." We have already argued that the use of the designation 'irreversible' in these quotes is a distraction. The substantive point, as his Fig. 1 (again on the same page) makes clear, is that these processes are between non-equilibrium states including their initial and terminating states. This denial of our achievability hypothesis has profound consequences. It effectively asserts that a thermodynamic system with a space of equilibrium states can never be said to be in any of these states. Or at least, if by some remarkable circumstances we encounter it in an equilibrium state, any processes effected by the control variables will force it to leave the state and ever after to wander in a wilderness of (undefined) non-equilibrium 'states'. This strikes at the heart of what is meant by an equilibrium state and a process in ordinary thermodynamics. It also has a further difficulty in the present context, where closeness to the curve $\mathcal{L}\left(\mathbf{x}_{0}, \mathbf{x}_{1}\right)$ is an issue. For the sequence of processes (3) defined by the sequence of points $\mathcal{S}_{m}\left(\mathcal{L}\left(\mathbf{x}_{0}, \mathbf{x}_{1}\right)\right)$ on $\mathcal{L}\left(\mathbf{x}_{0}, \mathbf{x}_{1}\right)$ closeness is simply defined by increasing $m$ and decreasing the maximum distance (in some well-defined metric) between any two points of the sequence. In terms of the delimiting process where closeness is between non-equilibrium and equilibrium states there is no corresponding metric.

In Sect. 7 of his paper Norton lists eleven attempts to solve the problem of equilibrium processes all of which, in his view, "are [either] incomplete or [...] include provisions that contradict one another, so that the characterization overall is a contradiction when read literally." The last of these, that of "iterated equilibria', approximates most closely to our own proposal. For this Norton references a number of authors of which O'Connell and Haile (2005) can most usefully be studied.

\subsection{Iterated Equilibria}

O'Connell and Haile (op. cit. Sect. 1.3) define $\Delta$ to be the net driving force acting on a system during a process. ${ }^{28}$ They then define $\delta$ as the "differential analogue of $\Delta$ " which produces a "differential process", which is their definition of a "quasi-static process". A finite process is then understood as the "stringing together [of] a sequence of quasi-static steps". From an equilibrium state the differential driving forces are used to take a step and then the system is allowed to relax back to equilibrium. Repeating this process many times generates "a sequence of points that represent a process path on a state diagram". For the curve $\mathcal{L}\left(\mathbf{x}_{0}, \mathbf{x}_{1}\right)$ this picture is essentially that given by the sequence of processes (3), where the set of points $\mathcal{S}_{m}\left(\mathcal{L}\left(\mathbf{x}_{0}, \mathbf{x}_{1}\right)\right.$ on the curve are close enough so that their separations can be understood as represented by differentials in the state variables. At this point there is a further discussion, which will not detain us, arguing that this quasi-static process along the curve is not necessarily reversible. The salient point is that this picture is one of a sequence of process between 'differentially spaced' equilibrium points on the curve. It is thus of a 'differentially spaced' member of the set of sequences of processes which we used, as shown in Fig. 1, to define the equilibrium process $\mathcal{L}\left(\mathbf{x}_{0}, \mathbf{x}_{1} \mid \mathcal{S}\right)$ demarcated by $\mathcal{L}\left(\mathbf{x}_{0}, \mathbf{x}_{1}\right)$.

The problem identified by Norton in this approach is the one with which we are now familiar, that "no single jump [of this string of quasi-static processes] can complete, if it is to terminate in an equilibrium state." And this, at its heart, arises from a fundamental difference in the standpoint of this paper, as described in Sect. 1 and summarized at the beginning of this section, and that of Norton with regard to the nature of thermodynamics. A difference which is often highlighted by the task of writing about or teaching classical/ordinary thermodynamics. There the temptation, even in quite formal accounts, is to appeal to

\footnotetext{
${ }^{28}$ They divide this into an external and internal part. The former can be understood as the effect of the control variables and the latter as the sum of forces generated within the system during the process, which decay during the leave-it-alone stage referred to in our hypothesis of achievability.
} 
the underlying microstructure. If such references are of an informal kind they can, of course, be of heuristic value. However, they can lead to problems.

An interesting example of this appears on page 17 of the otherwise very formal account of Lieb and Yngvason (1999). They observe that, for $\mathbf{x}, \boldsymbol{x}^{\prime} \in \Xi$, "when $\mathbf{x} \stackrel{\text { }}{\prec} \mathbf{x}^{\prime}$ and also $\mathbf{x}^{\prime} \stackrel{\AA}{\prec} \mathbf{x},[\ldots]$ the state change can only be realized in an idealized sense, for it will take an infinitely long time to achieve in the manner described;" that is to say using the mechanism for implementing an adiabatic process set down by Lieb and Yngvason and given above. Given that the very notion of the adiabatic accessibility $\boldsymbol{x}^{\mathrm{A}} \prec \boldsymbol{x}^{\prime}$, as used by Lieb and Yngvason, inhabits the fictitious world of ordinary thermodynamics, it is by no means obvious that $\boldsymbol{x}^{\mathrm{A}} \prec \boldsymbol{x}^{\prime}$ and $\boldsymbol{x}^{\prime} \stackrel{\text { A }}{\prec} \boldsymbol{x}$ cannot both apply without the need for an infinitely long time of implementation. Or more precisely, do adiabatic processes implementing $\boldsymbol{x} \rightarrow \boldsymbol{x}^{\prime}$ and $\boldsymbol{x}^{\prime} \rightarrow \boldsymbol{x}$ both take an infinite amount of time to complete, or just one of them in virtue of the existence of the other? If the latter is intended it is presumable because the state $\boldsymbol{x}$, which is the initial point of $\boldsymbol{x} \rightarrow \boldsymbol{x}^{\prime}$, is impossible to attain exactly in finite time as the destination of $\mathbf{x}^{\prime} \rightarrow \boldsymbol{x}$. But, if that is the case, it applies to all processes, and we are back to the situation discussed about, where the system never inhabits an equilibrium state. That there is a minefield here appears to be conceded by Lieb and Yngvason when they propose to avoid "this kind of discussion" by taking the definition of adiabatic accessibility "as given", and this is the spirit in which they proceed in the rest of their paper.

\section{Conclusions}

The problem of the oxymoronic nature of equilibrium processes is a problem within the structure of ordinary thermodynamics. There is an inherent conflict for a traditionally-understood equilibrium process between the role of the control variables as the only means of altering the equilibrium state of the system and the assumption that they cause the system to be disturbed from equilibrium. Our suggested solution to this problem based on sets of sequences of processes demarcated by a curve of equilibrium states remains firmly within the corpus of ordinary thermodynamics. The alternative solution proposed by Norton based on sets of non-equilibrium processes delimited by the curve of equilibrium states strays beyond the boundaries of ordinary thermodynamics and raises the problems we have discussed in the previous section.

The motivation of this work is the same as that of Norton, that is to repair in some way that part of the standard account of classical thermodynamics which uses the logically contradictory idea of an equilibrium process. He concludes (Norton, op. cit. p. 44) that the problem this presents is not "irresolvable" and offers his analysis "as a serviceable resolution". We offer ours in the same spirit.

\section{Acknowledgments}

I am very grateful to Roman Frigg and Reimer Kühn for reading and commenting on earlier versions of this work. I should also like to thank the reviewers for their constructive criticisms.

\section{References}

Boyling, J. B., 1972. An axiomatic approach to classical thermodynamics. Proc. Roy. Soc. A 329, 35-70.

Brown, H. R., Uffink, J., 2001. The origins of time-asymmetry in thermodynamics: the minus first law. Stud. Hist. Phil. Mod. Phys., 32, 525-538.

Buchdahl, H. A., 1966. The Concepts of Classical Thermodynamics. C.U.P. London.

Callen, H. B., 1985. Thermodynamics and an Introduction to Thermostatics, 2nd Edition. Wiley, New York.

Carathéodory, C., 1909. Untersuchungen über die Grundlagen der Thermodynamik. Mathematische Annalen 67, 355-386, page references given to the English translation: Investigation into the Foundations of Thermodynamics, by J. Kestin in The Second Law of Thermodynamics, Benchmark Papers on Energy, Vol. 5, (Ed. J. Kestin), Dowden, Hutchinson and Ross, 1976, pp. 229-256.

Cooper, J. L. B., 1967. The foundations of thermodynamics. J. Math. An. App. 17, $172-193$.

Duhem, P., 1902. Thermodynamique et Chimie: Leçons Élèmentaires à L'Usage des Chimistes. Librairie Scientifique A. Hermann: Paris.

Giles, R., 1964. Mathematical Foundations of Thermodynamics. Pergamon Press, Oxford. 
Joule, J. P., 1850. On the mechanical equivalent of heat. Proc. Roy. Soc. London 140, 61-82.

Landsberg, P. T., 1956. Foundations of thermodynamics. Rev. Mod. Phys. 28, 363-392.

Landsberg, P. T., 1961. Thermodynamics. Interscience, New York, London.

Lavis, D. A., 2015. Equilibrium Statistical Mechanics of Lattice Systems. Springer, Berlin, Heidelberg, New York.

Lebon, G., Jou, D., Casas-Vázquez, J., 2008. Understanding Non-Equilibrium Thermodynamics. Springer, Berlin, Heidelberg, New York.

Lieb, E. H., Yngvason, J., 1998. A guide to entropy and the second law of thermodynamics. Notices of the Amer. Math. Soc. $45,571-581$

Lieb, E. H., Yngvason, J., 1999. The physics and mathematics of the second law of thermodynamics. Phys. Rep. $310,1-96$.

Lieb, E. H., Yngvason, J., April 2000. A fresh look at entropy and the second law of thermodynamics. Physics Today 53, 32-37.

MacDonald, A., 1995. A new statement of the second law of thermodynamics. Am. J. Phys. 63, $1122-1127$.

Norton, J. D., 2016. The impossible process: thermodynamic reversibility. Stud. Hist. Phil. Mod. Phys. 55, 43-61.

O'Connell, J. P., Haile, J. M., 2005. Thermodynamics: Fundamentals for Applications. C.U.P. London.

Pippard, A. B., 1961. The Elements of Classical Thermodynamics. C. U. P. London.

Samiullah, M., 2007. What is a reversible process? Am. J. Phys. 75, 608-609.

Uffink, J., 2001. Bluff your way in the second law of thermodynamics. Stud. Hist. Phil. Mod. Phys. 32, 305-394.

Wallace, D., 2014. Thermodynamics as control theory. Entropy 16, 699-725.

Zemansky, M. W., 1968. Heat and Thermodynamics, 5th Edition. McGraw-Hill, New York. 